\title{
AN OVERVIEW OF THE APS 352-MHZ RF SYSTEMS
}

\author{
A. Nassiri, A. Cours, A. Grelick, D. Horan, Y.W. Kang, R.L. Kustom, T. L. Smith, and J. Song \\ Advanced Photon Source, Argonne National Laboratory \\ 9700 South Cass Avenue, Argonne, Illinois 60439 USA
}

Abstract

The Advanced Photon Source (APS) is a 7-GeV full energy positron storage ring for generating synchrotron radiation with an injector. The booster synchrotron rf system consists of a single 1-MW klystron which drives four five-cell cavities at $352 \mathrm{MHz}$. The storage ring cavities consist of four groups of four single cells powered by two 1-MW klystrons for 100-mA operation. An overview of the operation of the APS 352-MHz if systems is presented.

\section{INTRODUCTION}

The Advanced Photon Source (APS) at Argonne National Laboratory is a national user facility for synchrotron x-ray research constructed by the U. S. Department of Energy. It is a third-generation synchrotron radiation source specifically designed to accommodate insertion devices and operates beamlines from bending magnet radiation sources. The storage ring is designed to operate at 7 $\mathrm{GeV}$ with a full energy positron injector. The injector booster synchrotron consists of a single 1-MW klystron which drives four five-cell cavities at $352 \mathrm{MHz}$. These cavities are identical to those used in the ESRF and LEP storage rings [1].

The if transmitters in the storage ring are nearly identical to those of the booster synchrotron. The storage ring cavities consist of four groups of four single cells, powered by two 1-MW klystrons for $100-\mathrm{mA}$ operation. Two additional 1-MW transmitters are added to the existing system to support the ultimate design goal of $300 \mathrm{~mA}$ at $7 \mathrm{GeV}$. At least three transmitters are needed for $300-\mathrm{mA}$ operation, affording one hot spare for 300-mA operation.

For reliable and continuous operation of the booster synchrotron and the storage ring rf systems, the five if transmitters can be reconfigured to afford redundancy in operation using high-power WR-2300 waveguide switches [2]. An overview of the APS 352-MHz rf systems are presented.

\section{BOOSTER SYNCHROTRON}

\section{I Overview}

Four LEP-type five cell cavities are powered by a single 1-MW klystron (Thomson 2089). The power is split by waveguide hybrids with terminations on the fourth port going into the $250-\mathrm{kW}$ water-cooled resistive loads. The cavities are grouped in two pairs, each pair located symmetrically on opposite sides of the booster synchrotron ring. Since the klystron for the booster is located near one pair of the cavities (on the extraction side of the synchrotron ring), a motorized waveguide phase shifter is used in the 150-meter waveguide run to the cavities on the injec- tion side of the synchrotron ring. This phase shifter is used to compensate for any temperature-related changes in the length of the waveguide. The shunt impedance of each cavity is $55.3 \mathrm{M} \Omega$ with peak fundamental mode cavity dissipation of $475 \mathrm{~kW}$.

Injection rf voltage is about $100 \mathrm{kV}$, enough to capture the $450-\mathrm{MeV}$ positron beam. Energy gain per turn is 16 $\mathrm{keV}$. At the extraction energy of $7 \mathrm{GeV}$, the synchrotron radiation is $6.38 \mathrm{MeV} /$ turn and the rf voltage per turn is $10.4 \mathrm{MV}$, requiring about $480 \mathrm{~kW}$ power from the klystron.

\subsection{Low-Level $R f$}

The low-level system generates the rf signal to drive a klystron amplifier. The synchrotron signal generator is phase locked to the storage ring master oscillator. The storage ring if signal is transmitted to the synchrotron signal generator over phase stabilized cable [3].

\subsubsection{Tuners}

The second and fourth cells of each of the booster 5cell cavities have piston-type tuners. The cavity tuner port has an $11.5-\mathrm{cm}$ diameter and a $6.0-\mathrm{cm}$ travel. This results in a frequency tuning range of $500 \mathrm{kHz}$ to compensate for beam loading and temperature effects. By adjusting these two plungers both cavity tuning and amplitude balance in the cells are maintained. A feedback system monitors the phase difference in the cavity and adjusts the tuners in tandem to keep the cavity at the resonant frequency with $\pm 0.1^{\circ}$ under if heating. Also, the field amplitude in each of the two cells are detected and the tuners are adjusted deferentially to balance the cell voltage to within $\pm 1 \mathrm{~dB}$.

\subsubsection{Timing and Phasing}

The cavities are not a multiple wavelength apart; therefore, the waveguide feed between each pair of cavities is adjusted to 144 degrees so the beam sees the correct accelerating voltage. The sum signal is phased by the opposite amount to ensure proper combining. The cavities on the symmetrically opposite sides of the synchrotron are adjusted to a multiple of an rf wavelength by the motorized waveguide phase shifter so that proper accelerating phase is maintained in all four cavities.

\subsection{Beam Loading}

The booster synchrotron accelerates a single bunch (natural bunch length at $7 \mathrm{GeV}$ is $76 \mathrm{ps}$ ) of $4.7 \mathrm{~mA}$ (average beam current). The beam-induced voltage in each cell is $47 \mathrm{kV}$ or $940 \mathrm{kV}$ induced in all four 5-cell cavities. At injection, the synchrotron radiation loss is small, and for an energy gain per turn of $16 \mathrm{keV}$, the synchronous phase angle is 9.2 degrees. For a $100-\mathrm{kV}$ ring voltage, the detun- 


\section{DISCLAIMER}

This report was prepared as an account of work sponsored by an agency of the United States Government. Neither the United States Government nor any agency thereof, nor any of their employees, make any warranty, express or implied, or assumes any legal hability or responsibility for the accuracy, completeness, or usefulness of any information, apparatus, product, or process disclosed, or represents that its use would not infringe privately owned rights. Reference herein to any specific commercial product, process, or service by trade name, trademark, manufacturer, or otherwise does not necessarily constitute or imply its endorsement, recommendation, or favoring by the United States Government or any agency thereof. The views and opinions of authors expressed herein do not necessarily state or reflect those of the United States Government or any agency thereof. 


\section{DISCLAMIIR}

Portions of this document may be illegible in electronic image produets. Images are produced from the best available original document. 
ing angle is 83 degrees. In this case the vavity voltage can be increased to several hundred kilovolts to make the detuning and the beam injection tuning transient smaller. As the beam energy increases, radiation losses increase to $6.38 \mathrm{MeV} / \mathrm{turn}$ at $7 \mathrm{GeV}$ and the of voltage increases to $10.4 \mathrm{MV}$ with a synchronous phase angle of 37.5 degrees.

A set of measurements were made to characterize the effects of beam loading and other rt-related longitudinal beam instability in the booster synchrotron. The first measurement was made of the booster synchrotron tune as a function of time (using an HP vector signal analyzer to monitor the signal from a pickup electrode). A spectrograph of this measurement is shown in Figure 1.

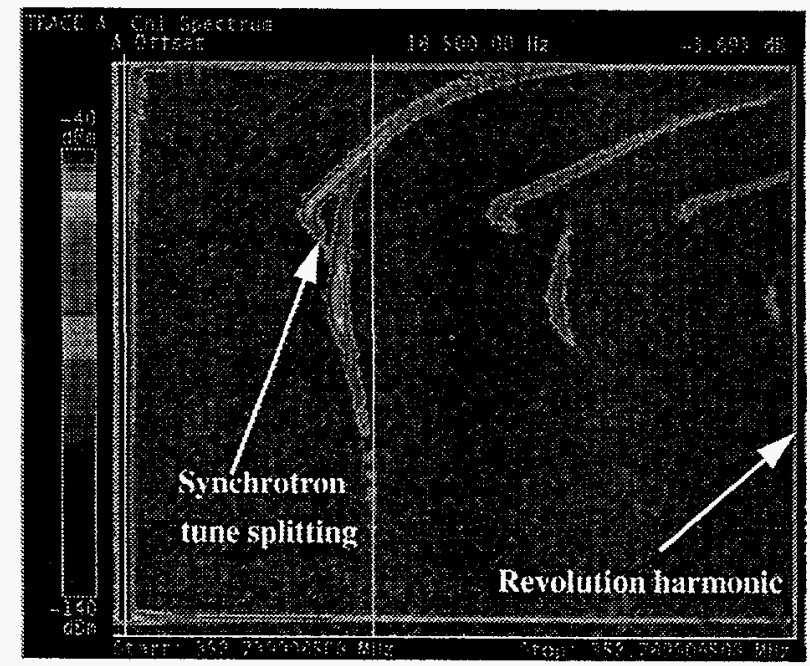

Figure 1: Spectrograph of the booster synchrotron tune vs. time (energy) for a full ramp. The duration of the spectrograph extends over the entire $223 \mathrm{~ms}$ of the ramp from $400 \mathrm{MeV}$ to $7 \mathrm{GeV}$.

The splitting of the synchrotron tune line partly up the ramp is an indication of beam loading. A streak camera was also used to study the longitudinal bunch distribution as a function of time during the booster ramp cycle. An image of the longitudinal bunch distribution is shown in Figure 2. The time scale is $100 \mathrm{~ms}$ horizontal and $1.5 \mathrm{~ns}$ in the vertical directions. This clearly shows the beam blow up (splitting of the bunch) during a beam loading episode. This happened approximately at the same time as observed on the splitting of the tune line in Figure 1.

The effect of beam loading was compensated by detuning all four cavities to about $20 \%$ of reflected power. Figure 3 shows the streak camera image of the longitudinal bunch distribution where the initial beam loading in the cavities is compensated.

\section{STORAGE RING RF}

\subsection{Overview}

In the current $100-\mathrm{mA}$ (positron beam) operation of the APS storage ring. two 1-MW klystrons (Thomson 2089) are used to provide of power to sixteen cavities.

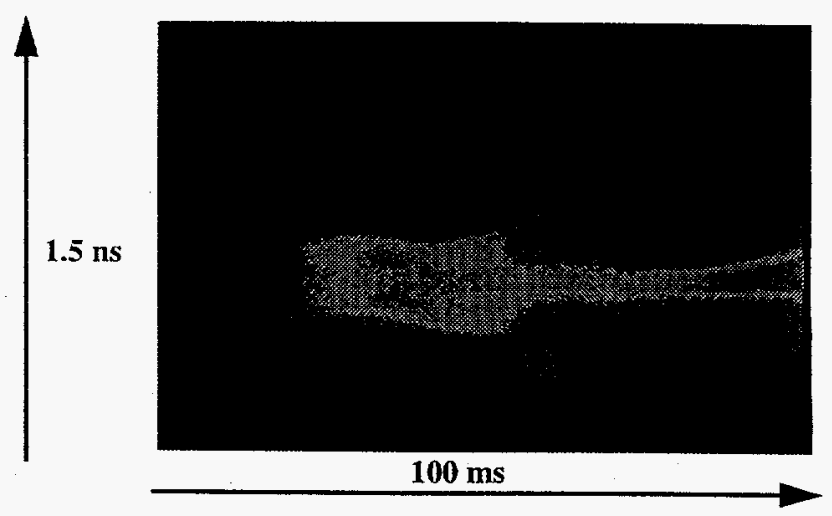

Figure 2: Longitudinal bunch distribution vs. time in the booster during a beam loading episode.

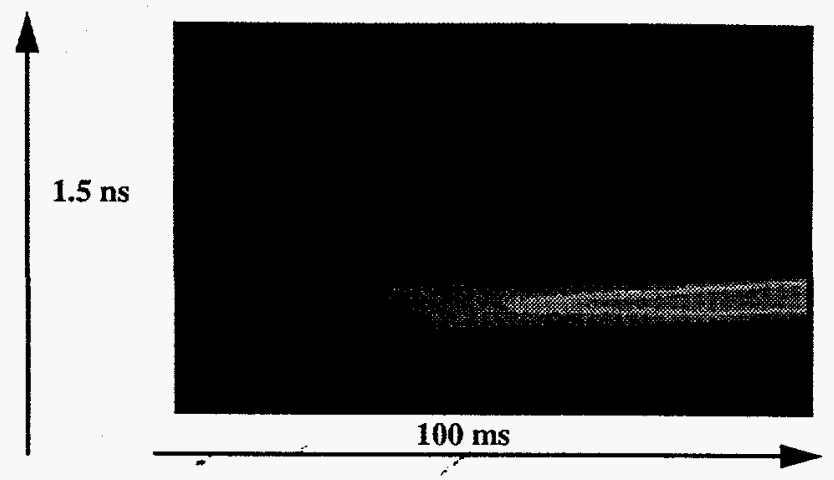

Figure 3: Longitudinal bunch distribution vs. time (initial beam loading is compensated).

Each klystron is powered and controlled by a $95-\mathrm{kV}, 20-\mathrm{A}$ DC power supply. These power supplies provide a high level of protection for the klystrons by using a combination of fast and slow interlock circuits and a very fast crowbar switch. Each power supply has a remote interface over an IEEE 488 bus to a remote computer control.

The sixteen cavities are divided into a group of four, with one group of cavities per rf sector. The cavities in each rf sector are uniformly spaced.

\subsection{Low-Level Rf}

The low-level if system for the storage ring klystron consists of two phase loops, one nested within the other; an AGC loop to provide constant rf drive to the klystron; slow and fast interlock systems which disable of drive in the event of a fault; and a 200-watt amplifier to drive the klystron. The internal phase loop removes phase jitter in the klystron output caused by high-voltage power supply (HVPS) ripple, and the second phase loop removes any residual phase jitter caused by the power supply and any phase distortion caused by other sources including beamcavity interaction. The interlock system removes rf drive at the milliwatt level in the event of a fault occurrence such as incorrect cavity temperature or water flow, vacuum in the cavities, resistive water load temperature and flow, or arcs in the waveguide, particularly at the klystron and cavity windows. The cavity low-level rf system consists of a 
phase loop around each cavity, maintaining proper phase between the forward power and field probe output. Phase errors are fed to the input of a stepping motor controller that moves a piston tuner to keep the cavity at resonance. Envelope detectors are used throughout the system to measure if power and to determine gap voltage.

\subsection{Rf controllaw}

To maintain the if gap voltage in the cavities, a software feedback program for collecting and processing data is interfaced to hardware through EPICS [4]. The rf controllaw is an application of sddscontrollaw [5] which performs simple feedback on process variables (PVs). Basically, a set of readback process variables is regulated to a set of control process variables to maintain if gap voltage. In the present if configuration each klystron powers a set of eight cavities in two separate rf sectors of the storage ring. There is one sddscontrollaw for each klystron. The output quantities are the probe power readbacks from the eight cavities. The control quantity for each sddscontrollaw process is the klystron mod anode voltage. Since there are eight output PVs and one control PV for each sddscontrollaw process, the gain matrix is a $1 \times 8$ matrix. The readback PVs are the cavity probe power measurements. The sum of the probe powers is kept constant. The individual probe power values are not mathematically constrained to be held constant; they may change individually due to sudden detuning of cavities or unequal coupling constants. The cavities probe powers are sampled as fast as possible. This allows faster response to beam loading transients when a beam dump occurs. The hardware interface to EPICS limits the probe powers reading to a $2-\mathrm{Hz}$ rate.

\subsection{Reconfigurable High-Power Rf}

To improve system reliability and availability, the APS 352-MHz rf systems have been configured to allow two to four klystrons to power the storage ring and one klystron to power or allow operation of the booster with one of the storage ring klystrons using high-power WR2300 waveguide switches. A quadrature hybrid combines two klystron outputs with two $45^{\circ}$ phase shifters at each output of the hybrid. Phase shifters in the hybrid outputs and three waveguide switches are used to choose any one of the twelve possible configurations. This system has been tested for mode selection and interlocks [2].

\subsection{Higher-Order Modes (HOMs)}

Longitudinal coupled-bunch (CB) instability was observed in the APS storage ring. This instability was found to depend on the bunch fill pattern as well as on the beam intensity. The beam spectrum exhibited a coupledbunch signature for different symmetric bunch fill patterns with a nominal beam current of $100 \mathrm{~mA}$. The beam fluctuations exhibited two periodicities, which were found to be correlated with the rf cavity temperatures. This intensitydependent collective effect is driven by long-range wake- fields such as those produced in the excitation by the beam of high-Q HOMs in the if accelerating cavities. To suppress the CB instability, the cavity water supply temperatures were increased to shift HOMs out of resonance with the beam [6].

Measurements of the beam-excited if cavity HOM spectra for different bunch patterns in the storage ring suggest that the $1210-\mathrm{MHz}$ mode is involved in the observed CB instability. Spectra were measured using E-type probes in the rf cavities [7]. To damp the HOMs in the storage ring of cavities, coaxial higher-order-mode dampers with high-pass filters are being designed and tested. These are E-type and H-type probe dampers with minimal deQing at the fundamental frequency [8].

\subsection{Beam Loading}

Computer simulation of the storage ring if system for the nominal $100 \mathrm{~mA}$ operation (assuming a total gap voltage of $8 \mathrm{MV}$ ) with optimal detuned cavities (i.e., zero loading angle) shows that the Robinson stability criterion is satisfied. The Robinson stability criterion is met for the ultimate goal of $300 \mathrm{~mA}$ operation, with a total ring voltage of $12 \mathrm{MV}$ and a cavity detuning angle of -42 degrees [9]. For the APS storage ring cavity this amounts to a -17 $\mathrm{kHz}$ detuning from the no-beam resonance condition to the fully loaded condition for the $300-\mathrm{mA}$ beam operation.

\section{ACKNOWLEDGMENTS}

We would like to thank K. C. Harkay, A. Lumpkin, and $B$. Yang for valuable discussions and assistance with the beam loading measurements of the booster synchrotron if cavities; L. Emery for implementing the controllaw software for the storage ring rf system; and J. N. Galayda for his support. This work is supported by the U. S. Department of Energy, Office of Basic Sciences, under Contract No. W-31-109-ENG-38.

\section{REFERENCES}

[1] J. Stepp, J. Bridges, "General Overview of the APS Low-Level RF Control System," Proc. of the 1993 Particle Accelerator Conference, p. 1157 (1993).

[2] Y. W. Kang et al.,"Reconfigurable High-Power RF System in the APS," these proceedings.

[3] D. Horan et al., "A Divide-Down RF Source Generation System for the Advanced Photon Source RF Systems," these proceedings.

[4] L. R. Dalesio et al., "EPICS Architecture," Proc. of ICALEPCS '91, Tsukuba-Ibaraki, Japan, KEK Proce. 92-15, 278-282, (1992).

[5] L. Emery, "Commissioning Software Tools at the Advanced Photon Source," Proc. of the 1995 Particle Accelerator Conference, p. 2238 (1996).

[6] K. C. Harkay et al., "Compensation of Longitudinal CoupledBunch Instability in the Advanced Photon Source Storage Ring," these proceedings.

[7] J. Song et al., "Higher-Order Modes of the Storage Ring RF Cavities," these proceedings.

[8] Y. W. Kang et al., "Coaxial Higher-Order Mode Damper Employing a High-Pass Filter," these proceedings.

[9] C. Schwartz et al., "Simulation of the APS Storage-Ring RF Accelerating System," these proceedings. 